\title{
Advancements in the treatment of pediatric acute leukemia and brain tumor - continuous efforts for $100 \%$ cure
}

Hee Young Ju, MD, Che Ry Hong, MD, Hee Young Shin, MD, PhD

Department of Pediatrics, Cancer Research Institute, Seoul National University College of Medicine, Seoul, Korea

Treatment outcomes of pediatric cancers have improved greatly with the development of improved treatment protocols, new drugs, and better supportive measures, resulting in overall survival rates greater than $70 \%$. Survival rates are highest in acute lymphoblastic leukemia, reaching more than $90 \%$, owing to risk-based treatment through multicenter clinical trials and protocols developed to prevent central nervous system relapse and testicular relapse in boys. New drugs including clofarabine and nelarabine are currently being evaluated in clinical trials, and other targeted agents are continuously being developed. Chimeric antigen receptor-modified $\mathrm{T}$ cells are now attracting interest for the treatment of recurrent or refractory disease. Stem cell transplantation is still the most effective treatment for pediatric acute myeloid leukemia (AML). However, in order to reduce treatmentrelated death after stem cell transplantation, there is need for improved treatments. New drugs and targeted agents are also needed for improved outcome of AML. Surgery and radiation therapy have been the mainstay for brain tumor treatment. However, chemotherapy is becoming more important for patients who are not eligible for radiotherapy owing to age. Stem cell transplant as a means of high dose chemotherapy and stem cell rescue is a new treatment modality and is often repeated for improved survival. Drugs such as temozolomide are new chemotherapeutic options. In order to achieve $100 \%$ cure in children with pediatric cancer, every possible treatment modality and effort should be considered.

Key words: Pediatrics, Neoplasms, Survival rate, Leukemia, Brain neoplasms

\section{Introduction}

Since the 1960s, there have been incremental improvements in treatment options for pediatric cancers. These improvements were fundamental for the advancement of the field of medical oncology, and led to the development of the following important concepts for cancer treatment; combination chemotherapy, multimodal treatment, riskbased treatment, and neo-adjuvant chemotherapy. Based on these treatment concepts, the 5-year survival rate of pediatric cancer has increased to up to $85 \%$ (Table 1$)^{1)}$. This advancement was mediated by improvements in prophylaxis and treatment of infection and bleeding, which had been the main cause of death in pediatric cancer patients in the early 1970s. Nevertheless, some patients remain refractory to treatment, and successful treatment after relapse is still difficult to achieve. This review focuses on the recent advancements to overcome refractory or relapsed disease, as well as current advances in supportive care.
Corresponding author: Hee Young Shin, MD, PhD Department of Pediatrics, Cancer Research Institute, Seoul National University College of Medicine, 103 Daehak-ro, Jongno-gu, Seoul 110-799, Korea Tel: +82-2-2072-2917

Fax: +82-2-3675-0999

E-mail: hyshin@snu.ac.kr

Received: 30 June, 2014

Accepted: 14 August, 2014

Copyright $(\subset 2014$ by The Korean Pediatric Society

This is an open-access article distributed under the terms of the Creative Commons Attribution NonCommercial License (http://creativecommons.org/ licenses/by-nc/3.0/) which permits unrestricted noncommercial use, distribution, and reproduction in any medium, provided the original work is properly cited. 


\section{Acute lymphoblastic leukemia}

The current 5-year survival rate of childhood acute lymphoblastic leukemia (ALL) exceeds 85\% in developed countries. This improvement has been achieved by the optimal use of antileukemic agents, dose modification, improved drug combinations, optimized administration schedules, and better supportive care. As most patients with newly diagnosed ALL are cured, current studies are focused on curing relapsed or refractory patients, as well as the reduction of long-term treatment-related morbidity for survivors.

New agents are gradually applied in relapsed pediatric ALL patients (Table 2). Clofarabine is a drug approved by the United States Food and Drug Administration for pediatric patients prior to adult patients. It has shown promising results for relapsed pediatric patients when used as a single agent or as combination therapy along with standard chemotherapy agents as cyclopho-

\begin{tabular}{lc}
\multicolumn{2}{l}{ Table 1. Five-year survival rates for pediatric cancer ${ }^{1)}$} \\
\hline Disease & Overall survival (\%) $^{2}$ \\
\hline ALL & $85-90$ \\
AML & $60-70$ \\
Brain tumor & $62-80$ \\
Neuroblastoma & $64-88$ \\
Rhabdomyosarcoma & $46-64$ \\
Wilms tumor & 92 \\
Osteosarcoma & 67 \\
Ewing sarcoma & $49-76$
\end{tabular}

ALL, acute lymphoblastic leukemia; AML, acute myeloid leukemia.

Table 2. New agents for pediatric acute lymphoblastic leukemia

\begin{tabular}{lll}
\hline Class & \multicolumn{1}{c}{ Agent } & Disease \\
\hline Monoclonal antibodies & Alemtuzumab & ALL \\
& Gemtuzumab ozogamicin & AML \\
& Blinatumomab & ALL \\
Nucleoside analogues & Clofarabine & ALL, AML \\
& Nelarabine & T-ALL \\
BCR-ABL inhibitors & Imatinib & PH+ ALL \\
& Dasatinib & PH+ ALL \\
FLT3 inhibitors & Sorafenib & $\mathrm{AML}$ \\
& Lestaurtinib & $\mathrm{ALL}$ \\
& AC220 & $\mathrm{ALL}, \mathrm{AML}$ \\
JAK inhibitors & Ruxolitinib & $\mathrm{ALL}, \mathrm{AML}$ \\
mTOR inhibitors & Rapamycin & $\mathrm{ALL}$ \\
& Everolimus & $\mathrm{ALL}$ \\
Proteosome Inhibitors & Bortezomib & $\mathrm{ALL}, \mathrm{AML}$ \\
\hline
\end{tabular}

ALL, acute lymphoblastic leukemia; AML, acute myeloid leukemia; JAK, Janus kinase; PH+, Philadelphia chromosome positive; T-ALL, T-cell ALL; FLT3, FMSlike tyrosine kinase 3; mTOR, mechanistic target of rapamycin. sphamide or etoposide $e^{2)}$. Clofarabine is currently being investigated in a clinical trial by the Children's Oncology Group (COG) in combination with cyclophosphamide and etoposide as front-line therapy for very high-risk pediatric ALL patients (clinical trial number NCT00408005). A multicenter clinical trial examining clofarabine in relapsed ALL children was initiated this year in Korea with cooperation of the Health Insurance Review Et Assessment Service and the Korean Society of Pediatric Hematology Oncology. If this clinical trial achieves greater than a 30\% 3-year survival rate, then clofarabine will be covered by medical insurance. This is the first risk-sharing model for new drugs in Korea. Nelarabine is a particularly active nucleoside analogue for T-cell disease. A phase I study of nelarabine as a single agent for pediatric refractory malignancies demonstrated an overall response rate of $42 \%{ }^{33}$. Nelarabine is currently under investigation in a COG phase III trial for newly diagnosed T-cell ALL and lymphoblastic lymphoma (NCT00408005).

Tyrosine kinases are constitutively activated in a subgroup of leukemia and are therefore attractive targets for therapeutic intervention. The tyrosine kinase inhibitor imatinib has been shown to greatly improve survival when added to chemotherapy for children with Philadelphia chromosome-positive ALL, resulting in a 3-year event-free survival (EFS) of $80 \%{ }^{4}$. Following this promising result, hematopoietic stem cell transplantation (HSCT) was not performed for first complete remission (CR) in Philadelphia chromosome-positive ALL when minimal residual disease (MRD) was negative in a study performed at St. Jude hospital ${ }^{5}$. Dasatinib, a second generation tyrosine kinase inhibitor is 325 times more potent than imatinib for inhibiting the BCR-ABL ${ }^{5)}$, and is able to cross the bloodbrain barrier ${ }^{6}$. Continuous use of dasatinib in combination with standard chemotherapy is currently being investigated in a clinical trial for children newly diagnosed with Philadelphiachromosome positive ALL (NCT01460160).

Another actively studied field in pediatric cancer is cellular therapy. Multiple studies of cellular therapy for pediatric cancers are currently being performed with promising results ${ }^{6}$. Chimeric antigen receptor (CAR)-modified T cells with specificity for CD19 have shown promising results in the treatment of pediatric ALL ${ }^{6)}$. Natural killer cells are lymphocytes of the innate immune system that can kill target cancer cells by ligand-mediated interactions with various killer inhibitor receptors (KIRs). Practical application of cellular therapy will greatly expand treatment options for pediatric $\mathrm{ALL}^{7)}$.

\section{Acute myeloid leukemia}

Acute myeloid leukemia (AML) accounts for 15\%-20\% of pediatric leukemia, and the overall survival (OS) of pediatric 
AML is approximately $60 \%-70 \%$. The treatment outcomes of pediatric AML have improved with effective use of chemotherapeutics, improvement of supportive care, and risk-group stratification. However, this progress has plateaued, with survival rates substantially lower than those for ALL, where OS exceeds $80 \%$. Current steps to further improve AML treatment include monitoring of MRD, and tailoring treatment with novel therapeutics.

In ALL, measurement of MRD by leukemia-specific polymerase chain reaction (PCR)-based quantitative techniques has emerged as a very powerful prognostic factor, and riskdirected therapy has resulted in the improvement of ALL EFS approaching 90\%. The heterogeneity of AML has made MRD application difficult, but the use of quantitative PCR-technology has eliminated these difficulties. The AML02 multicenter clinical trial adopted MRD to establish the final risk assignment and to determine treatment for pediatric $\mathrm{AML}^{8)}$. According to this study, the use of chemotherapy and HSCT according to MRD-based risk grouping could improve outcomes in patients with childhood AML. Furthermore, the authors suggested that more sensitive flow-cytometric methods based on more than four-color analysis should improve the sensitivity of MRD testing and may improve the identification of patients who are likely to have poor early treatment response.

Novel therapeutic agents are emerging as new options for pediatric AML. Novel cytarabine derivatives (cladribine, fludarabine) have shown promising results. Unlike cytarabine, cladribine and fludarabine inhibit both DNA polymerase and ribonucleotide reductase, thereby depleting deoxynucleotide pools. Cladribine has shown encouraging results in combination with cytarabine ${ }^{9)}$, topotecan ${ }^{10)}$, and idarubicin ${ }^{11}$. In particular, acute monoblastic leukemia (FAB M5) was shown to be most sensitive to cladribine. Fludarabine in combination with cytarabine with or without anthracycline is widely used as a reinduction regimen for children with relapsed $\mathrm{AML}^{12,13}$. Clofarabine is a structural hybrid of cladribine and fludarabine, designed to have better efficacy and improved stability. For childhood AML, an ongoing randomized phase II study is comparing end-of-induction MRD for children treated with clofarabine and cytarabine versus conventional ADE therapy (cytarabine, daunorubicin, etoposide) (NCT 00703820).

Gemtuzumab ozogamicin (G0), a humanized anti-CD33 monoclonal antibody conjugated to the cytotoxic compound $\mathrm{N}$-acetyl-gamma-calicheamicin dimethyl hydrazide, has shown improved response when added to cytarabine in relapsed/ refractory AML ${ }^{14)}$. The AML02 clinical trial showed tolerability and efficacy of G0 when incorporated in the induction chemotherapy treatment protocol.

FMS-like tyrosine kinase 3 (FLT3) inhibitors are emerging as a new targeted therapeutics in pediatric AML. The first generation
FLT3 inhibitors such as CEP-701 (lestaurtinib) and PKC412 (midostaurin) are nonselective compounds with potent antiFLT3 activity. Second generation FLT3 inhibitors such as AC220 (quizartinib), designed with increased potency and selectivity for FLT3 are currently being evaluated in early-phase clinical trials.

A key issue for pediatric cancer patients has been reducing exposure to cardiotoxic drugs. Liposomal daunorubicin causes less cardiotoxicity than the nonliposomal daunorubicin formulation by preferential release of daunorubicin in tumor cells $^{15)}$. The liposomal daunorubicin option can decrease late events related to chemotherapy. Cardioprotective drugs such as cardioxane (dexrazoxane) help to greatly reduce the cardiac toxicity from anthracycline in long-term survivors ${ }^{16)}$. Reducing the mortality and morbidity of chemotherapy-associated complications such as cardiac toxicity is becoming increasingly important when treating childhood malignancies.

Allogeneic stem cell transplantation (SCT) is no longer considered the first choice of treatment for first CR in low risk AML. In patients with low-risk or intermediate-risk AML without a matched-related donor, treatment-related mortality and morbidity may exceed the benefit. However, most oncologists consider allogeneic SCT in relapsed AML. More studies are needed to refine the indications of allogeneic SCT in pediatric AML.

For patients who undergo allogeneic SCT, prophylaxis of complications is important for improving the OS. One example is the prevention of veno-occlusive disease (VOD) with lipoprostaglandin E1 (PGE1). Incidence of VOD ranges from 11\% to $31 \%$ of pediatric SCT, but the mortality rate has been reported to be up to $50 \%{ }^{17-20)}$. Because of the high mortality rate, the prevention of VOD is critical. The effectiveness of PGE1 has been reported for prevention of $\mathrm{VOD}^{211}$, and lipo-PGE1 was suggested as a more potent drug with fewer side effects when compared to PGE1 ${ }^{22)}$. Recent studies have shown the effectiveness of lipoPGE1 for prevention of VOD in pediatric $\mathrm{SCT}^{23,24)}$. Future efforts should be focused on reducing the transplantation-related mortality.

\section{Brain tumor}

Medulloblastoma is the most common pediatric malignant brain tumor, accounting for 20\% of all cases ${ }^{25}$. Medulloblastoma can be classified into the following subtypes: classic, desmoplastic, large cell, anaplastic, medullomyoblastoma, and melanotic medulloblastoma. Recent data suggest that the desmoplastic subtype has improved prognosis in young infants ${ }^{26}$, but the large cell and anaplastic types have poor prognosis ${ }^{27}$. The mainstay treatment of medulloblastoma/primitive neuroectodermal tumor (PNET) is surgical resection; craniospinal 
radiotherapy is also required for improved outcome. Recent medulloblastoma trials focus on reduced-dose craniospinal radiation and adjuvant chemotherapy for average-risk patients. Good survival rates were reported using sequential high-dose chemotherapy with autologous stem cell rescue following craniospinal radiation ${ }^{28)}$. The survival of children under 3 years of age with PNET remains poor. For children younger than 3 years of age who cannot receive radiotherapy because of possible cognitive dysfunction, high-dose chemotherapy with autologous stem cell rescue may be the optimal treatment. Recent genomic studies have identified distinct subtypes of medulloblastoma, which are defined by sonic hedgehog (SHH), Wnt, or other pathway activation. SHH antagonists and targeted agents for ErbB family receptors (erlotinib, lapatinib) are being explored. Development of targeted therapy is expected to expand treatment options for medulloblastoma.

High-grade glioma (HGG), including anaplastic astrocytoma and glioblastoma multiforme, together with diffuse intrinsic pontine glioma (DIPG), constitute approximately 25\% of pediatric brain tumors. HGGs and DIPGs have poor survival outcomes with fewer than 10\% of children with GBM or DIPG surviving 2 years after diagnosis ${ }^{29)}$. Treatment of HGG consists of surgery followed by radiation therapy combined with chemotherapy. Studies examining higher doses and different schedules of radiation fractionation to brainstem gliomas have shown no improvement in responses; standard radiation is still recommended because clinical improvement and tumor response is observed in about 30\% of patients. In adult glioblastoma multiforme, adjuvant temozolomide has provided significant improvements in survival. However, in the 2011 COG study, results with temozolomide for HGGs and DIPG were similar to those obtained in the CCG-945 phase III trial with lomustine and vincristine following radiotherapy ${ }^{30}$. A phase I trial with lomustine and temozolomide showed improved EFS, but no difference in $0 S^{31}$. Multicenter prospective trials are needed to further improve the survival rates of HGG patients.

\section{Advancements in supportive care}

Current guidelines for the treatment of infection include empirical antibiotic therapy to cover the most likely pathogens such as gram-negative bacilli including Pseudomonas aeruginosa and viridans group streptococci in high-risk febrile neutropenia patients ${ }^{32}$. Monotherapy with an antipseudomonal beta-lactam or a carbapenem is recommended as the initial treatment, and prompt administration of antibiotics has been shown to improve outcome of febrile neutropenia ${ }^{33}$. Antifungal prophylaxis in cancer patients has been shown to lower the rate of invasive fungal infections. Patients at high risk of invasive fungal infection include those who need transplantation from an unrelated donor or a partially matched related donor, for the treatment of malignancy, bone marrow failure syndrome, or congenital immunodeficiency ${ }^{34-36)}$. Children with AML have a high incidence of invasive fungal infection, both in newly diagnosed and relapsed patients ${ }^{37,38}$. In contrast, ALL patients are considered to be at high risk for invasive fungal infection only when they relapse ${ }^{39}$. For these high-risk patients, agents used for antifungal prophylaxis include fluconazole. Fluconazole prevents candida infection but lacks activity against Aspergillus spp, which is the second-most common cause of invasive fungal infection in pediatric cancer patients. Thus, echinocandins such as caspofungin could be utilized to cover both molds and candida; however, further study is needed to evaluate the efficacy in children. The usage of colony-stimulating factors contributes to the control of infection in cancer patients. Routine use of granulocyte colony-stimulation factors decreased the incidence of febrile neutropenia and duration of hospitalization and may decrease delays in subsequent chemotherapy, but it does not lead to improvement in $\mathrm{OS}^{40-43)}$.

Prophylactic platelet transfusions have contributed to lowering the risk of bleeding in pediatric cancer patients. Prophylactic platelet transfusions are the standard of care for adult patients with hypoplastic thrombocytopenia after receiving chemotherapy or radiation ${ }^{44)}$. A randomized controlled study of pediatric cancer patients showed that patients who received prophylactic platelet transfusions had shorter number of days with bleeding ${ }^{45}$, and another study revealed that prophylactic platelet transfusion resulted in fewer bleeding episodes ${ }^{46)}$. Another study showed that platelet counts of $<10^{5} / \mu \mathrm{L}$ was independently associated with an increased probability of traumatic bloody lumbar puncture ${ }^{47)}$, which was associated with increased risk of relapse ${ }^{48}$. Platelet transfusion had been in the form of platelet concentrate before the use of platelet pheresis; small children could not receive more than 2 units of platelet concentrate owing to the problem of volume overloading and bleeding could not be sufficiently controlled. Since the development of pheresis machine, small children can now receive more than 6 units of platelet at once without the risk of volume overloading so that bleeding can be controlled. Patients 0-5 years of age have been shown to have higher clinically significant bleeding incidence at PLADO (prophylactic platelet dose trial) analysis when compared to older patients ${ }^{49)}$; however, there was insufficient power to determine differences of bleeding outcomes. Future randomized controlled trials should be designed to compare platelet transfusion regimens. 


\section{Conclusions}

Survival rates of childhood cancers have continued to improve over the past decade, particularly for leukemia patients. Future efforts at improving survival in pediatric cancer patients should focus on relapsed or refractory disease. In particular, the application of targeted agents or cell therapies should be the subject of future studies of relapsed leukemia. Some pediatric and adolescent solid tumors have shown no definite advancement in survival over the past 10 to 20 years; these include Ewing sarcoma, osteosarcoma, and rhabdomyosarcoma. Similarly, survival improvements have been modest for pediatric brain tumors over the past 10 to 15 years for most age groups1). There is a need for increased focus on developing novel therapies that are based on understanding the cellular pathways promoting tumor growth and survival.

\section{Conflict of interest}

No potential conflict of interest relevant to this article was reported.

\section{Acknowledgments}

This study was supported by a grant from the National RetD program for Cancer Control, Ministry of Health \& Welfare, Republic of Korea (1420250).

\section{References}

1. Smith MA, Seibel NL, Altekruse SF, Ries LA, Melbert DL, O'Leary $\mathrm{M}$, et al. Outcomes for children and adolescents with cancer: challenges for the twenty-first century. J Clin Oncol 2010;28:262534.

2. Hijiya N, Gaynon P, Barry E, Silverman L, Thomson B, Chu R, et al. A multi-center phase I study of clofarabine, etoposide and cyclophosphamide in combination in pediatric patients with refractory or relapsed acute leukemia. Leukemia 2009;23:2259-64.

3. Kurtzberg J, Ernst TJ, Keating MJ, Gandhi V, Hodge JP, Kisor DF, et al. Phase I study of 506 U78 administered on a consecutive 5-day schedule in children and adults with refractory hematologic malignancies. J Clin Oncol 2005;23:3396-403.

4. Schultz KR, Bowman WP, Aledo A, Slayton WB, Sather H, Devidas $\mathrm{M}$, et al. Improved early event-free survival with imatinib in Philadelphia chromosome-positive acute lymphoblastic leukemia: a children's oncology group study. J Clin Oncol 2009;27:5175-81.

5. Jeha S, Coustan-Smith E, Pei D, Sandlund JT, Rubnitz JE, Howard SC, et al. Impact of tyrosine kinase inhibitors on minimal residual disease and outcome in childhood Philadelphia chromosomepositive acute lymphoblastic leukemia. Cancer 2014;120:1514-9.

6. Porkka K, Koskenvesa P, Lundan T, Rimpilainen J, Mustjoki S,
Smykla R, et al. Dasatinib crosses the blood-brain barrier and is an efficient therapy for central nervous system Philadelphia chromosome-positive leukemia. Blood 2008;112:1005-12.

7. Bhojwani D, Pui CH. Relapsed childhood acute lymphoblastic leukaemia. Lancet Oncol 2013;14:e205-17.

8. Rubnitz JE, Inaba H, Dahl G, Ribeiro RC, Bowman WP, Taub J, et al. Minimal residual disease-directed therapy for childhood acute myeloid leukaemia: results of the AML02 multicentre trial. Lancet Oncol 2010;11:543-52.

9. Rubnitz JE, Crews KR, Pounds S, Yang S, Campana D, Gandhi VV, et al. Combination of cladribine and cytarabine is effective for childhood acute myeloid leukemia: results of the St Jude AML97 trial. Leukemia 2009;23:1410-6.

10. Inaba H, Stewart CF, Crews KR, Yang S, Pounds S, Pui CH, et al. Combination of cladribine plus topotecan for recurrent or refractory pediatric acute myeloid leukemia. Cancer 2010;116:98105.

11. Chaleff S, Hurwitz CA, Chang M, Dahl G, Alonzo TA, Weinstein H. Phase II study of 2-chlorodeoxyadenosine plus idarubicin for children with acute myeloid leukaemia in first relapse: a paediatric oncology group study. Br J Haematol 2012;156:649-55.

12. Kaspers GJ, Zimmermann M, Reinhardt D, Gibson BE, Tamminga $\mathrm{RY}$, Aleinikova 0 , et al. Improved outcome in pediatric relapsed acute myeloid leukemia: results of a randomized trial on liposomal daunorubicin by the International BFM Study Group. J Clin Oncol 2013;31:599-607.

13. Gorman MF, Ji L, Ko RH, Barnette P, Bostrom B, Hutchinson $\mathrm{R}$, et al. Outcome for children treated for relapsed or refractory acute myelogenous leukemia (rAML): a Therapeutic Advances in Childhood Leukemia (TACL) Consortium study. Pediatr Blood Cancer 2010;55:421-9.

14. Brethon B, Yakouben K, Oudot C, Boutard P, Bruno B, Jerome C, et al. Efficacy of fractionated gemtuzumab ozogamicin combined with cytarabine in advanced childhood myeloid leukaemia. Br J Haematol 2008;143:541-7.

15. Gabizon A, Martin F. Polyethylene glycol-coated (pegylated) liposomal doxorubicin. Rationale for use in solid tumours. Drugs 1997;54 Suppl 4:15-21.

16. Lipshultz SE, Scully RE, Lipsitz SR, Sallan SE, Silverman LB, Miller TL, et al. Assessment of dexrazoxane as a cardioprotectant in doxorubicin-treated children with high-risk acute lymphoblastic leukaemia: long-term follow-up of a prospective, randomised, multicentre trial. Lancet Oncol 2010;11:950-61.

17. Cesaro S, Pillon M, Talenti E, Toffolutti T, Calore E, Tridello G, et al. A prospective survey on incidence, risk factors and therapy of hepatic veno-occlusive disease in children after hematopoietic stem cell transplantation. Haematologica 2005;90:1396-404.

18. Barker CC, Butzner JD, Anderson RA, Brant R, Sauve RS. Incidence, survival and risk factors for the development of venoocclusive disease in pediatric hematopoietic stem cell transplant recipients. Bone Marrow Transplant 2003;32:79-87.

19. Cacchione A, LeMaitre A, Couanet DV, Benhamou E, Amoroso L, Simonnard N, et al. Risk factors for hepatic veno-occlusive disease: a retrospective unicentric study in 116 children autografted after a high-dose BU-thiotepa regimen. Bone Marrow Transplant 2008;42:449-54.

20. Ozkaynak MF, Weinberg K, Kohn D, Sender L, Parkman R, Lenarsky C. Hepatic veno-occlusive disease post-bone marrow transplantation in children conditioned with busulfan and cyclophosphamide: incidence, risk factors, and clinical outcome. Bone Marrow Transplant 1991;7:467-74.

21. Gluckman E, Jolivet I, Scrobohaci ML, Devergie A, Traineau R, 
Bourdeau-Esperou H, et al. Use of prostaglandin E1 for prevention of liver veno-occlusive disease in leukaemic patients treated by allogeneic bone marrow transplantation. Br J Haematol 1990; 74:277-81.

22. Mizushima Y, Yanagawa A, Hoshi K. Prostaglandin E1 is more effective, when incorporated in lipid microspheres, for treatment of peripheral vascular diseases in man. J Pharm Pharmacol 1983;35:666-7.

23. Lee SH, Yoo KH, Sung KW, Koo HH, Kwon YJ, Kwon MM, et al. Hepatic veno-occlusive disease in children after hematopoietic stem cell transplantation: incidence, risk factors, and outcome. Bone Marrow Transplant 2010;45:1287-93.

24. Song JS, Seo JJ, Moon HN, Ghim T, Im HJ. Prophylactic lowdose heparin or prostaglandin E1 may prevent severe venoocclusive disease of the liver after allogeneic hematopoietic stem cell transplantation in Korean children. J Korean Med Sci 2006;21:897-903.

25. Pollack IF. Multidisciplinary management of childhood brain tumors: a review of outcomes, recent advances, and challenges. J Neurosurg Pediatr 2011;8:135-48.

26. McManamy CS, Lamont JM, Taylor RE, Cole M, Pearson AD, Clifford SC, et al. Morphophenotypic variation predicts clinical behavior in childhood non-desmoplastic medulloblastomas. J Neuropathol Exp Neurol 2003;62:627-32.

27. von Hoff K, Hartmann W, von Bueren A0, Gerber NU, Grotzer MA, Pietsch T, et al. Large cell/anaplastic medulloblastoma: outcome according to myc status, histopathological, and clinical risk factors. Pediatr Blood Cancer 2010;54:369-76.

28. Gajjar A, Chintagumpala M, Ashley D, Kellie S, Kun LE, Merchant TE, et al. Risk-adapted craniospinal radiotherapy followed by high-dose chemotherapy and stem-cell rescue in children with newly diagnosed medulloblastoma (St Jude Medulloblastoma-96): long-term results from a prospective, multicentre trial. Lancet Oncol 2006;7:813-20.

29. Gajjar A, Packer RJ, Foreman NK, Cohen K, Haas-Kogan D, Merchant TE, et al. Children's Oncology Group's 2013 blueprint for research: central nervous system tumors. Pediatr Blood Cancer 2013;60:1022-6.

30. Cohen KJ, Pollack IF, Zhou T, Buxton A, Holmes EJ, Burger PC, et al. Temozolomide in the treatment of high-grade gliomas in children: a report from the Children's Oncology Group. Neuro Oncol 2011;13:317-23.

31. Jakacki RI, Yates A, Blaney SM, Zhou T, Timmerman R, Ingle AM, et al. A phase I trial of temozolomide and lomustine in newly diagnosed high-grade gliomas of childhood. Neuro Oncol 2008;10:569-76.

32. Lehrnbecher T, Phillips R, Alexander S, Alvaro F, Carlesse F, Fisher $\mathrm{B}$, et al. Guideline for the management of fever and neutropenia in children with cancer and/or undergoing hematopoietic stem-cell transplantation. J Clin Oncol 2012;30:4427-38.

33. Fletcher M, Hodgkiss H, Zhang S, Browning R, Hadden C, Hoffman T, et al. Prompt administration of antibiotics is associated with improved outcomes in febrile neutropenia in children with cancer. Pediatr Blood Cancer 2013;60:1299-306.

34. Dvorak CC, Steinbach WJ, Brown JM, Agarwal R. Risks and outcomes of invasive fungal infections in pediatric patients undergoing allogeneic hematopoietic cell transplantation. Bone Marrow Transplant 2005;36:621-9.

35. Burgos A, Zaoutis TE, Dvorak CC, Hoffman JA, Knapp KM, Nania
JJ, et al. Pediatric invasive aspergillosis: a multicenter retrospective analysis of 139 contemporary cases. Pediatrics 2008;121:e128694.

36. Mikulska M, Raiola AM, Bruno B, Furfaro E, Van Lint MT, Bregante $\mathrm{S}$, et al. Risk factors for invasive aspergillosis and related mortality in recipients of allogeneic SCT from alternative donors: an analysis of 306 patients. Bone Marrow Transplant 2009;44:361-70.

37. Sung L, Gamis A, Alonzo TA, Buxton A, Britton K, DeswarteWallace J, et al. Infections and association with different intensity of chemotherapy in children with acute myeloid leukemia. Cancer 2009;115:1100-8.

38. Rosen GP, Nielsen K, Glenn S, Abelson J, Deville J, Moore TB. Invasive fungal infections in pediatric oncology patients: 11year experience at a single institution. J Pediatr Hematol Oncol 2005;27:135-40.

39. Afzal S, Ethier MC, Dupuis LL, Tang L, Punnett AS, Richardson SE, et al. Risk factors for infection-related outcomes during induction therapy for childhood acute lymphoblastic leukemia. Pediatr Infect Dis J 2009;28:1064-8.

40. Heath JA, Steinherz PG, Altman A, Sather H, Jhanwar S, Halpern $\mathrm{S}$, et al. Human granulocyte colony-stimulating factor in children with high-risk acute lymphoblastic leukemia: a Children's Cancer Group Study. J Clin Oncol 2003;21:1612-7.

41. Sung L, Nathan PC, Lange B, Beyene J, Buchanan GR. Prophylactic granulocyte colony-stimulating factor and granulocyte-macrophage colony-stimulating factor decrease febrile neutropenia after chemotherapy in children with cancer: a meta-analysis of randomized controlled trials. J Clin Oncol 2004;22:3350-6.

42. Pui CH, Boyett JM, Hughes WT, Rivera GK, Hancock ML, Sandlund JT, et al. Human granulocyte colony-stimulating factor after induction chemotherapy in children with acute lymphoblastic leukemia. N Engl J Med 1997;336:1781-7.

43. Ozkaynak MF, Krailo M, Chen Z, Feusner J. Randomized comparison of antibiotics with and without granulocyte colonystimulating factor in children with chemotherapy-induced febrile neutropenia: a report from the Children's Oncology Group. Pediatr Blood Cancer 2005;45:274-80.

44. Schiffer CA, Anderson KC, Bennett CL, Bernstein S, Elting LS, Goldsmith M, et al. Platelet transfusion for patients with cancer: clinical practice guidelines of the American Society of Clinical Oncology. J Clin Oncol 2001;19:1519-38.

45. Murphy S, Litwin S, Herring LM, Koch P, Remischovsky J, Donaldson $\mathrm{MH}$, et al. Indications for platelet transfusion in children with acute leukemia. Am J Hematol 1982;12:347-56.

46. Roy AJ, Jaffe N, Djerassi I. Prophylactic platelet transfusions in children with acute leukemia: a dose response study. Transfusion 1973;13:283-90.

47. Howard SC, Gajjar A, Ribeiro RC, Rivera GK, Rubnitz JE, Sandlund JT, et al. Safety of lumbar puncture for children with acute lymphoblastic leukemia and thrombocytopenia. JAMA 2000;284: 2222-4.

48. Gajjar A, Harrison PL, Sandlund JT, Rivera GK, Ribeiro RC, Rubnitz JE, et al. Traumatic lumbar puncture at diagnosis adversely affects outcome in childhood acute lymphoblastic leukemia. Blood 2000; 96:3381-4.

49. Josephson CD, Granger S, Assmann SF, Castillejo MI, Strauss RG, Slichter SJ, et al. Bleeding risks are higher in children versus adults given prophylactic platelet transfusions for treatment-induced hypoproliferative thrombocytopenia. Blood 2012;120:748-60. 